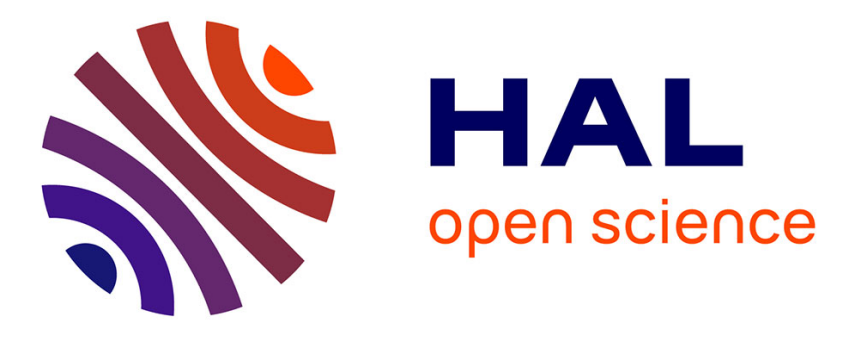

\title{
Cortical bone viscoelastic damping assessed with resonant ultrasound spectroscopy reflects porosity and mineral content
}

Fan Fan, Xiran Cai, Hélène Follet, Françoise Peyrin, Pascal Laugier, Haijun

Niu, Quentin Grimal

\section{To cite this version:}

Fan Fan, Xiran Cai, Hélène Follet, Françoise Peyrin, Pascal Laugier, et al.. Cortical bone viscoelastic damping assessed with resonant ultrasound spectroscopy reflects porosity and mineral content. Journal of the mechanical behavior of biomedical materials, 2021, 117, pp.104388. 10.1016/j.jmbbm.2021.104388 . hal-03210991

\section{HAL Id: hal-03210991 https: / hal.sorbonne-universite.fr/hal-03210991}

Submitted on 28 Apr 2021

HAL is a multi-disciplinary open access archive for the deposit and dissemination of scientific research documents, whether they are published or not. The documents may come from teaching and research institutions in France or abroad, or from public or private research centers.
L'archive ouverte pluridisciplinaire HAL, est destinée au dépôt et à la diffusion de documents scientifiques de niveau recherche, publiés ou non, émanant des établissements d'enseignement et de recherche français ou étrangers, des laboratoires publics ou privés. 


\title{
Cortical bone viscoelastic damping assessed with resonant ultrasound spectroscopy reflects porosity and mineral content
}

\author{
Fan Fan ${ }^{\mathrm{b}, \mathrm{a}, *}$, Xiran Cai ${ }^{\mathrm{b}, \mathrm{c}}$, Hélène Follet ${ }^{\mathrm{d}}$, Françoise Peyrin ${ }^{\mathrm{e}}$, Pascal Laugier $^{\mathrm{b}, \mathrm{a}}$, Haijun $\mathrm{Niu}^{\mathrm{a}}$, \\ Quentin Grimal ${ }^{\mathrm{b}}$ \\ ${ }^{a}$ Beijing Advanced Innovation Center for Biomedical Engineering, School of Biological Science and Medical Engineering, \\ Beihang University, 100083, Beijing, China \\ ${ }^{b}$ Sorbonne Université, INSERM UMR-S 1146, CNRS UMR 7371, Laboratoire d'Imagerie Biomédicale, F-75006, Paris, France \\ ${ }^{c}$ School of Information Science and Technology, ShanghaiTech University, 201210, Shanghai, China \\ ${ }^{d}$ Univ Lyon, Université Claude Bernard Lyon 1, INSERM, LYOS UMR 1033, F-69008, Lyon, France \\ ${ }^{e}$ Univ Lyon, INSA-Lyon, Université Claude Bernard Lyon 1, UJM-Saint Etienne, CNRS, Inserm, CREATIS UMR 5220, \\ U1206, F-69621, Lyon, France
}

\begin{abstract}
Viscoelasticity is an essential property of bone related to fragility, which is altered in aging and bone disease. Bone viscoelastic behavior is attributed to several mechanisms involving collagen and mineral properties, porosities, and bone hierarchical tissue organization. We aimed to assess the relationships between cortical bone viscoelastic damping measured with Resonant Ultrasound Spectroscopy (RUS), microstructural and compositional characteristics. We measured 52 bone specimens from the femur of 26 elderly human donors. RUS provided a shear damping coefficient at a frequency of the order of $150 \mathrm{kHz}$. The characteristics of the structure of the vascular pore network and tissue mineral density were measured using synchrotron radiation high-resolution computed tomography (SR- $\mu \mathrm{CT}$ ). Fourier transformed infrared microspectroscopy (FTIRM) was used to quantify mineral-to-organic phase ratio, mineral maturity, crystallinity, and collagen maturity. Cross-links were quantified from biochemistry. Viscoelastic damping was found to increase with vascular porosity $(r=0.68)$, to decrease with the degree of mineralization of the extravascular matrix $(r=-0.68)$, and was marginally affected by collagen. We built a multilinear model suggesting that when porosity is controlled, the variation of mineral content explains a small additional part of the variability of damping. The work supports the consideration of viscoelasticity measurement as a potential biomarker of fragility and provides a documentation of bone viscoelastic behavior and its determinants in a frequency range rarely investigated.
\end{abstract}

Keywords: Cortical bone, Damping, Quality factor, Resonant ultrasound spectroscopy, Porosity, Mineral content

\footnotetext{
${ }^{*}$ Corresponding author

Email address: fanfan@buaa.edu.cn (Fan Fan)
} 


\section{Introduction}

Viscoelasticity is an essential property of biological tissues [1]. For example, hard biological tissues, such as bone, exhibit creep and stress relaxation, rate dependent response to a dynamic loading, phase lag between stress and strain during oscillatory loading, and damping of elastic waves [2]. Bone viscoelastic behavior is attributed to several mechanisms [3] involving all scales from the nanoscale to the mesoscale, i.e. the scale of a few millimeters in cortical bone [4]. The collagen of the extracellular matrix is itself viscoelastic. The movement of fluids within the pores, heat flow under mechanical loading between heterogeneous regions such as osteons and lamellae, are some of the other mechanisms associated to the viscoelastic behavior.

Viscoelasticity of bone has been investigated by many authors motivated by the relationship between damping mechanisms and mechanical behavior beyond the elastic limit and related to bone fragility. Firstly, collagen was shown to have a profound effect on bone fragility, because changes in collagen content, or changes to collagen cross-linking, reduce the energy required to cause failure [5]. Secondly, bone strength [6, 7] and toughness [8] are rate dependent suggesting a role for viscous mechanisms. Thirdly, there is a close relationship between microdamage and viscoelasticity as loading cortical bone past the yield stress changes its viscoelastic properties [9]. Finally, the viscoelastic dissipation of energy at micron and sub-micron scales is related to crack initiation and propagation [10,11]. Bone material quality and fragility are multifaceted phenomena involving several scales and they cannot be fully captured from a single mechanical measurement. This has led several authors to suggest that the measurement of viscoelasticity could bring unique information related to fragility associated with bone disease [12-14] and tissue alteration during aging [15]. Yet, viscoelastic data is scarce in comparison to published data on elastic properties. In particular, the relationships between viscoelasticity, extravascular matrix composition, and microstructure are poorly documented.

Viscoelasticity has been assessed with a variety of techniques such as microscale measurement of creep with nanoindentation [15-17], mesoscale measurement of an oscillatory response in torsion (up to $50 \mathrm{kHz}$ ) [18], or 3-point bending with dynamic mechanical analyzers (in the range 1-20 Hz) [19]. In material science, another popular approach is to use resonant ultrasound spectroscopy (RUS), a technique to measure the anisotropic stiffness and viscoelastic damping assessed from the width of a resonance of a freely vibrating specimen [20-23]. In a viscoelastic material such as bone, the resonant peaks corresponding to the different eigenmodes of the measured specimen tend to overlap. It follows that RUS is essentially practicable to measure bone damping associated with the first eigenmode which is associated to a shear modulus [23, 24]. A typical cortical bone specimen for RUS measurements is a cuboid with longest dimension around 5 mm, having its first eigenfrequency around $150 \mathrm{kHz}$.

The purpose of the present work was to assess the relationships between cortical bone viscoelastic damping measured with RUS, tissue composition, porosity, and microstructure. We measured a collection of bone specimens from elderly human donors with RUS, providing a quality factor (equivalent to tan $\delta$ in torsion experiments), associated to a shear modulus, at a frequency of the order of $150 \mathrm{kHz}$. Vascular porosity and the degree of mineralization of bone were obtained from synchrotron radiation high-resolution computed tomography (SR- $\mu \mathrm{CT}$ ), cross-links were quantified from biochemistry, Fourier transformed infrared spectroscopy 
(FTIR) was used to quantify mineral-to-organic phase ratio, mineral maturity, crystallinity, and collagen maturity. Note that the relationship between elastic properties and these variables was previously reported in $[25]$.

The work provides a documentation of bone viscoelastic behavior and its determinants in a frequency range rarely investigated, complementing existing data. Comparison with the viscoelastic behavior at other frequencies may provide insight into the mechanics of bone viscoelasticity. While our results do not reflect the viscoelastic behavior of bone at the frequencies of physiological loading, they are of practical interest for some ultrasonic applications around $10^{5} \mathrm{~Hz}$ such as bone ultrasonic drilling, ultrasonic stimulation of bone healing and bone health assessment with guided waves.

\section{Materials and methods}

\subsection{Specimens}

We have used a collection of specimens from a previous study [25]. The preparation of specimens is briefly recalled here. Cortical bone specimens were harvested from the left femur of 26 human cadavers. The femurs were provided by the Départment Universitaire d'Anatomie Rockefeller (Lyon, France) through the French program on voluntary corpse donation to science. Among the donors, 14 were females and 12 were males $(50-95$ years old, $77.3 \pm 11.5$, mean \pm SD). As shown in Figure 1, in each of the lateral and medial anatomical quadrants, adjacent specimens (\# 1, \# 2 and \# 3) were prepared along the axial direction, to be measured by several techniques described below. All specimens were frozen and stored at $-20^{\circ} \mathrm{C}$ between tests. They were then slowly thawed and immersed in $0.9 \% \mathrm{NaCl}$ saline before testing to ensure full hydration [26]. The nominal size of the specimens \#1 used for RUS was $3 \times 4 \times 5 \mathrm{~mm}^{3}$ in radial $\left(x_{1}\right)$, circumferential $\left(x_{2}\right)$ and axial $\left(x_{3}\right)$ directions, respectively. These specific dimensions were chosen so as to maximize the size of the specimens while complying with the technical requirements of RUS [22]. Figure 2 is the three-dimensional rendering of the SR- $\mu \mathrm{CT}$ image of a bone specimen. Specimens \#1 used in RUS were kept hydrated prior to experiment and all RUS measurements were made on a fully hydrated specimen. After a first set of RUS measurements, specimens \#1 were defatted following a protocol which prevents the risk of infections and allows the specimen conservation at room temperature [26].

\subsection{RUS experiment}

Setup and signal processing methods dedicated to the RUS measurement of attenuative materials, extensively described elsewhere [22, 27], were used in this study. Briefly, the specimen \#1 was mounted on opposite corners between two shear wave transducers (V154RM, Panametrics, Waltham, MA, USA). The frequency response (vibration spectrum) was recorded using a vectorial network analyzer (Bode 100, Omicron Electronics GmbH, Klaus, Austria) and a broadband charge amplifier (HQA-15M-10T, Femto Messtechnik GmbH, Berlin, Germany). The frequency band of analysis was 100-700 kHz, including the first resonant frequency of the specimen. Six successive spectra were recorded for each specimen, with intermediate rotation (without unmounting) of the specimen by a small angle between each measurement to vary the relative amplitudes of 


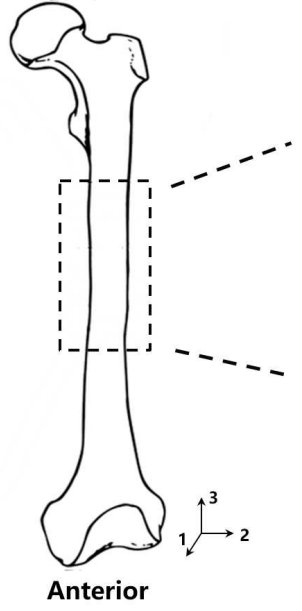

(a)

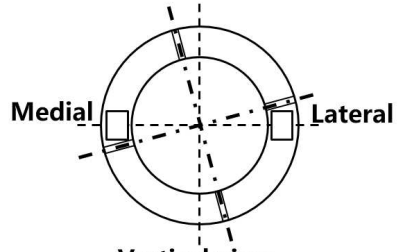

Vertical view

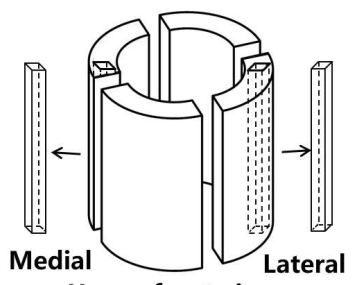

Upper-front view

(b)

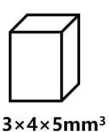

\#1
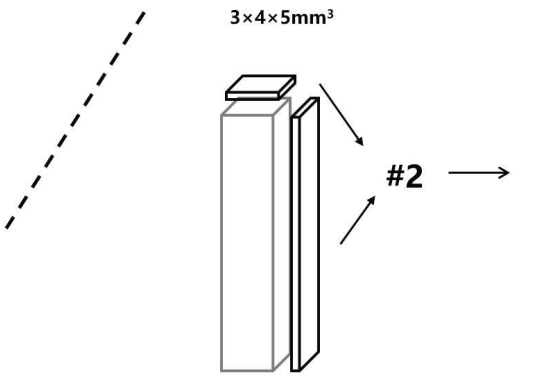

\#3

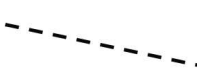

(1)

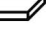

(c)

(d) RUS

Viscoelastic damping

(e) SR- $\mu C T$

Microstructure

\& DMB

(f) Biochemistry

Collagen cross-link

Figure 1: Specimen preparation procedure.(a) A cross-section of femoral bone at the mid-diaphysis was extracted. (b) The vertical and upper-front view of the cross section, which was then cut into 4 pieces (lateral, medial, anterior and posterior). Two of these pieces (lateral and medial) were then used. (c) 3 rectangular parallelepiped shaped specimens (set \#1, \#2 and \#3) were prepared along the axial direction at both the lateral and medial quadrants. (d) RUS measurements for bone viscoelastic damping. (e) SR- $\mu \mathrm{CT}$ scanning for Bone microstructural parameters and DMB. (f) Bone residues close-by \#2 after cutting for \#2 went to biochemistry experiments for the collagen and cross-links. The data experiments carried on \#2 were not shown in this work. (g) FTIRM tests for bone compositional information.

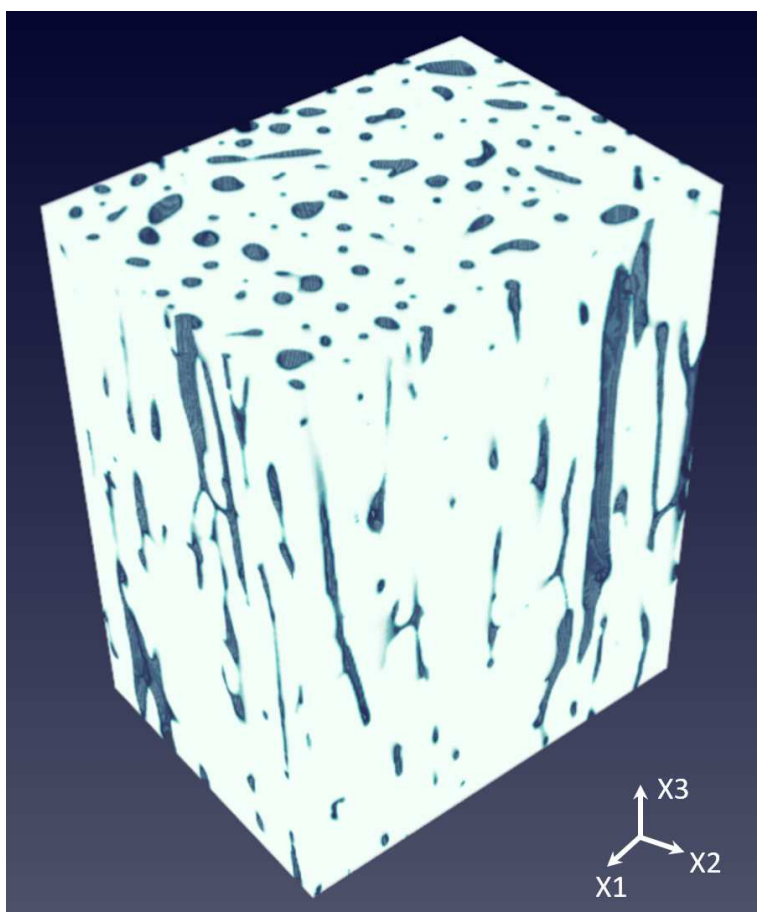

Figure 2: Three-dimensional rendering of the SR- $\mu \mathrm{CT}$ image of a bone specimen of approximate dimensions $3 \times 4 \times 5 \mathrm{~mm}^{3}$.

73 the excited resonant modes in order to maximize the number of detectable resonant frequencies (Fig. 3). A 74 selected portion of each complex spectrum can be fitted by a sum of $M$ Lorentzian line-shapes (each describing 
the behavior of a one degree-of-freedom mechanical resonator) :

$$
L(f)=\sum_{k=1}^{M} \frac{a_{k}}{\left(f_{k}^{2}-f^{2}\right)+i\left(f_{k} f / Q_{k}\right)}
$$

with $f$ the frequency, $a_{k}$ the complex amplitudes, $f_{k}$ the resonant frequencies and $Q_{k}$ the quality factors. The quality factor is related to the width of the resonant peak as $Q_{k} \sim f_{k} / \Delta f$, where $\Delta f$ is the -3db bandwidth (half-power bandwidth).

Upon combining the six spectra and fitting with Eq. (1), between 20 to 30 resonant frequencies $f_{k}$ were extracted for each specimen [22]. Frequencies $f_{k}$, which are nearly equal to the eigenfrequencies of the freely vibrating specimen, were then used to determine the coefficients $C_{i j}$ of the stiffness tensor [20,27]. These were obtained in a previous work presented in [25]. We used Voigt notation for the stiffness tensor and we assumed that bone is a transversely isotropic material (plane 1-2 is the plane of isotropy). As a consequence, $C_{11}=C_{22}, C_{13}=C_{23}, C_{44}=C_{55}$.

For the purpose of the present study, we specifically processed the first resonant peak (around $150 \mathrm{kHz}$ in Fig. 3) to assess shear mode damping. As explained in section 2.3, the shear damping coefficient $Q_{44}^{-1}$ (associated to elastic coefficient $C_{44}$ ) can be obtained from the quality factor of the first resonant mode $Q_{1}$ (defined in Eq. (1)). The quality factor $Q_{1}$ in each spectrum was obtained as follows :

- A portion of the spectrum (bandwidth) was manually selected, containing only the first peak $f_{1}$ (Case 1 , Fig. 4 left) or the two first peaks (Case 2, Fig. 4 right). Precisely, Case 1 corresponds to specimens with a well-isolated first resonant peak. For other specimens with first two relatively close resonant peaks (Case 2), we selected a portion of the spectrum with the first two peaks to account for the potential influence of the second resonance on the Lorentzian lineshape of the first peak. The distance between the two first peaks depends on the exact dimensions of the specimen and its elastic properties. Among the 52 specimens, 10 were in Case 1 and 42 were in Case 2. The effect on frequency and Quality factor determination of the bandwidth selection method is discussed further in Appendix A.

- To determine $Q_{1}$, Eq. (1) was fitted to the spectrum assuming $M=1$ (Case 1) or $M=2$ (Case 2) using a time domain estimation method based on a linear predictive filter (black dash line in Fig 5), followed by a frequency domain nonlinear optimization (black solid line) [22, 28].

Specimens were measured with RUS before defatting, after defatting, and finally after X-ray irradiation during SR- $\mu C T$.

\subsection{Calculation of material damping}

In this section, we present how the measured quality factor $Q_{1}$ was used to calculate shear damping coefficient $Q_{44}^{-1}$ following [23, 24]. Bone viscoelasticity was modeled by introducing the complex modulus:

$$
C_{i j}^{*}=C_{i j}+i C_{i j}^{\prime}=C_{i j}\left(1+i Q_{i j}^{-1}\right)
$$




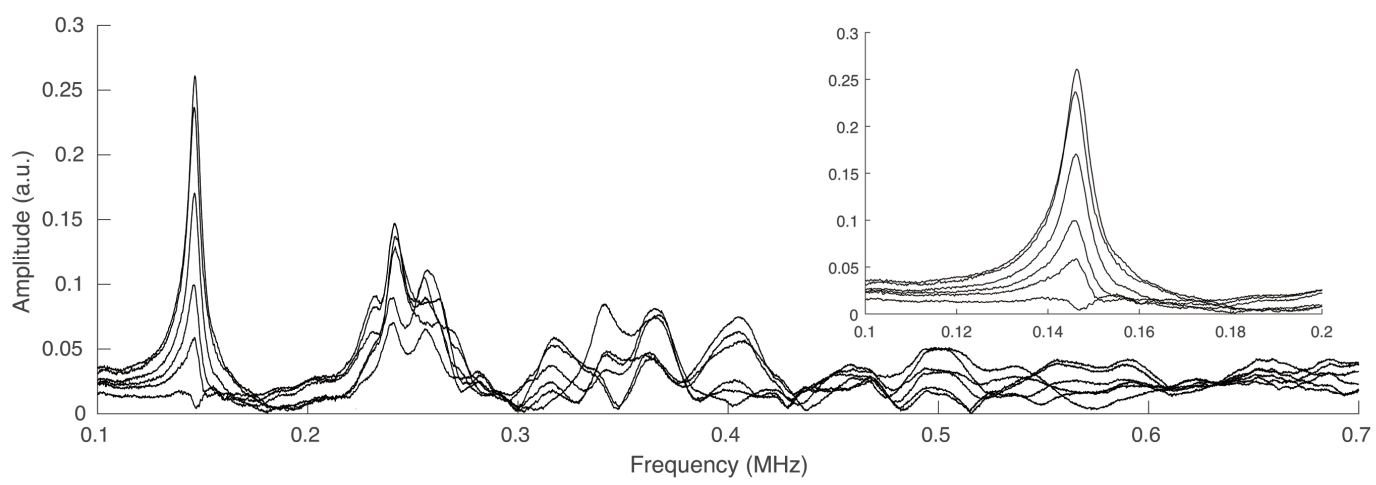

Figure 3: Typical set of spectra measured for a cortical bone specimen. The relative amplitudes of the resonant peaks vary as the specimen is rotated. The width of the first peak provided a measurement of damping. The inset figure is a zoom of in the frequency range of 100 to $200 \mathrm{kHz}$.
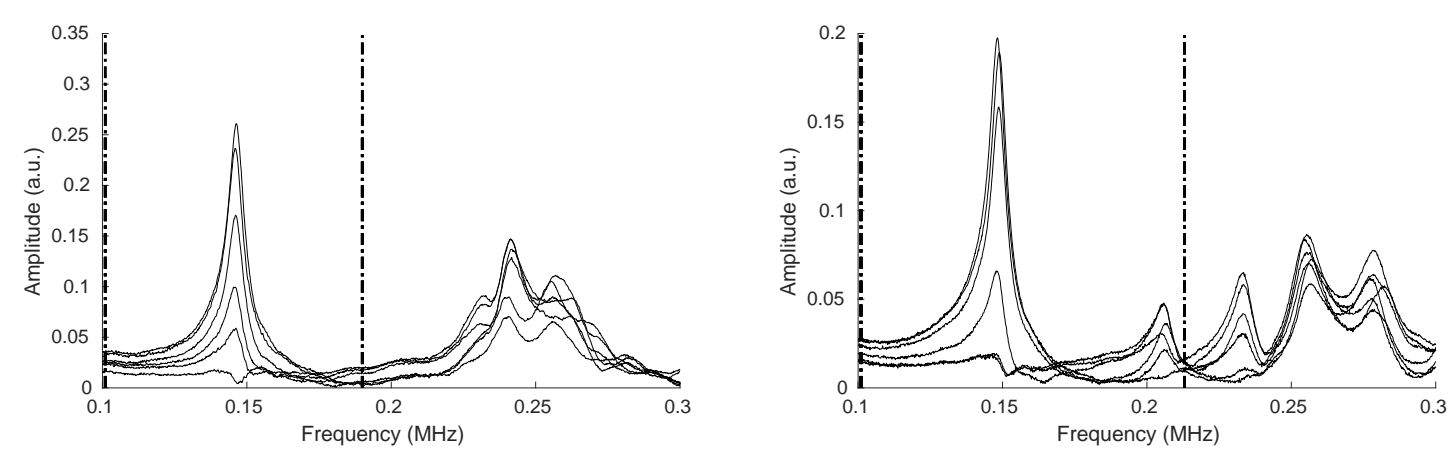

Figure 4: To calculate $Q_{1}$, a portion of the spectrum was selected between the two dash-dot lines. (left) Case 1: for specimens with a well-isolated first resonant peak, only the peak was selected. (right) Case 2: for other specimens, the first two peaks were selected together.
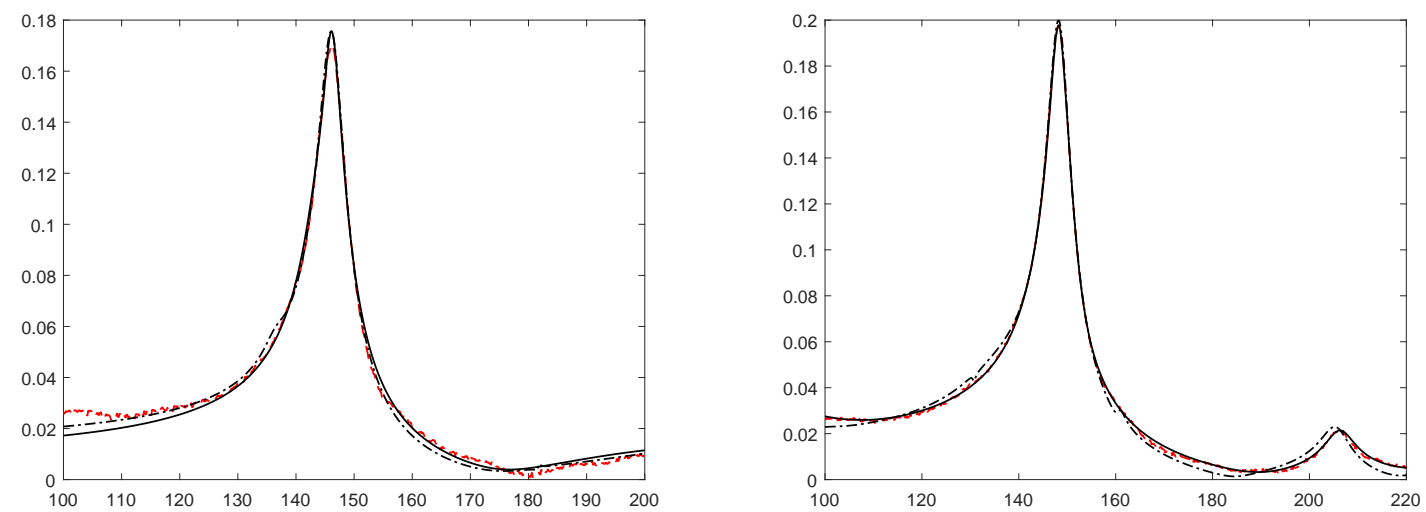

Figure 5: Illustration of the fit of the first peak with Eq. (1). Red dash lines show experimental frequency response of Case 1 (left) and Case 2 (right), frequency response reconstructed with a linear predictive filter (black dash line), and then followed by a frequency domain nonlinear optimization (black solid line).

where $C_{i j}$ and $C_{i j}^{\prime}$ are the stiffness coefficient and loss modulus, respectively, and $Q_{i j}^{-1}=\frac{C_{i j}^{\prime}}{C_{i j}}$. This definition of the complex modulus holds for a dynamic loading at a given frequency, and $C_{i j}$ and $C_{i j}^{\prime}$ are in general functions of frequency. Note that $Q_{i j}^{-1} \sim \tan \delta$, where $\tan \delta$, another popular way of reporting damping, is the phase shift between a harmonic loading and the mechanical response in a vibrational mechanical test [2]. 
In the present work, the complex modulus is evaluated using the first resonant peak centered at $f_{1}$ of each specimen.

In a RUS experiment, a series of resonant frequencies $f_{k}$ and Quality factors $Q_{k}$ can in principle be obtained from the resonant spectrum. These are related to the complex modulus through [29-31] :

$$
Q_{k}^{-1}=\sum_{i, j} \frac{2 C_{i j}}{f_{k}} \frac{\partial f_{k}}{\partial C_{i j}} Q_{i j}^{-1}
$$

where

$$
\sum_{i, j} \frac{2 C_{i j}}{f_{k}} \frac{\partial f_{k}}{\partial C_{i j}}=1 .
$$

The linear system of equations Eq. (3) between $Q_{i j}^{-1}$ and $Q_{k}^{-1}$ is derived with the assumption that $Q_{i j}^{-1} \ll 1$, which is in practice the case for cortical bone $[23,24]$. The coefficients of the linear system are the relative sensitivities of the eigenmode $k$ to the loss moduli $Q_{i j}$.

When the stiffness coefficients $C_{i j}$ are available, the system of equations (3) can be inverted to derive $Q_{i j}^{-1}$ from the measured $Q_{k}^{-1}$. In practice, quality factors $Q_{k}$ for $k=2,3, \cdots$ can hardly be estimated with a sufficient precision in high damping materials such as bone because of strong resonant peak overlapping. Furthermore, several relative sensitivity terms are small, so that the inversion is ill-conditioned and sensitive to errors in the measured $Q_{k}$. Here, we take advantage of the fact that:

1. The first resonant peak is well-separated from the subsequent peaks (Fig. 4) for all specimens. This is a result of the specific aspect ratio of the specimens which was selected so as to optimize the separation of low frequency resonances. As a consequence, $Q_{1}$ is estimated with a satisfactory precision ;

2. The terms

$$
\frac{2 C_{i j}}{f_{1}} \frac{\partial f_{1}}{\partial C_{i j}}
$$

for $i j \neq 44$ are relatively small.

Hence, the relationship between $Q_{1}^{-1}$ and $Q_{44}^{-1}$ simplifies to,

$$
Q_{1}^{-1} \approx \frac{2 C_{44}}{f_{1}} \frac{\partial f_{1}}{\partial C_{44}} Q_{44}^{-1}
$$

The quality of this approximation was checked by calculating the relative sensitivity $\frac{2 C_{44}}{f_{1}} \frac{\partial f_{1}}{\partial C_{44}}$ of the first eigenmode to the shear coefficient $C_{44}$ for the collection of specimens. We found that close to $90 \%$ of the value of $Q_{1}^{-1}$ is determined by $Q_{44}^{-1}$, i.e., $\frac{2 C_{44}}{f_{1}} \frac{\partial f_{1}}{\partial C_{44}}=89.7 \% \pm 2.0 \%$ (mean $\left.\pm \mathrm{SD}\right)$. From a physical perspective, the first eigenmode of the specimens is a pure shear mode involving mostly the shear modulus $C_{44}$ and corresponding damping $Q_{44}^{-1}[20]$.

\subsection{Microstructural, mineral and collagen variables}

In the present work, we use the same data set as [25] where the measurement protocols of the microstructural, collagen and mineral variables were extensively described. Briefly, (i) Fourier transform infrared mi- 
crospectroscopy (FTIRM) was used to measure collagen maturity (CollMat), mineral-to-organic ratio (MinOrga), mineral maturity (Minmat), carbonation, and crystallinity index (CryInd); (ii) biochemical measurements on hydrolyzates prepared from powdered demineralized bone residues provided the amount of enzymatic cross-links (DHLNL, HLNL, PYD and DPD), non-enzymatic cross-links (PEN), and the amount of collagen (Coll); (iii) SR- $\mu \mathrm{CT}$ (pixel size $6.5 \mu \mathrm{m}$ ) was used to determine vascular porosity (Ct.Po) and other microstructural variables (PoS/PoV, PoN, PoDm, PoSp, PoPf, ConnD and SMI), and the degree of mineralization of bone (DMB). The definitions of these variables are collected in Table 1 grouped as collagen, mineral, and microstructural variables.

\subsection{Data analysis}

Normality of the variables was evaluated using the Shapiro-Wilk test. One-way analysis of variance (ANOVA) and Wilcoxon test (for the variables failing the normality test) were performed to evaluate the differences of the data sets from lateral and medial anatomical quadrants. As some variables were not normally distributed, Spearman rank correlation coefficients between $Q_{44}^{-1}$ and each of the mineral, collagen, and microstructural variables were calculated.

The variables significantly correlated with $Q_{44}^{-1}$ were retained for stepwise multiple linear regression analyses. To highlight the relative importance of the explanatory variables in the model, all the variables except $Q_{44}^{-1}$ were normalized between -1 and 1 using the equation,

$$
\bar{x}=2 \frac{x-\min x}{\max x-\min x}-1,
$$

where $x$ is the variable to be normalized. The multiple linear regression analyses were firstly carried out in each group of variables (microstructure, mineral and collagen). Then, the most significant variable of each group was retained to find the optimal multiple linear regression model. Linear models were evaluated using the adjusted-r ${ }^{2}\left(\operatorname{Adj}-r^{2}\right)$ and root-mean-square-error (RMSE).

Note that for some variables which showed significant differences between lateral and medial specimens, the multiple linear regression analyses were carried out both on the data sets of lateral and medial specimens separately and the corresponding model are reported. If these variables were not retained as explanatory variables in the regression model, analyses were run again pooling the data sets from lateral and medial specimens.

Data were considered statistically significant for $p<0.05$. Statistical analyses were made using the Matlab 2017a Statistics Toolbox (Mathworks Inc., Natick, MA, USA).

We present below the statistical results for $Q_{44}^{-1}$ measured by RUS before defatting. We found that defatting or irradiation by X-ray for tomography imaging did not significantly modify $Q_{44}^{-1}$ (see Appendix B). 
Table 1: Microstructure, mineral and collagen variables and their definitions.

\begin{tabular}{|c|c|c|c|}
\hline Variable & Unit & Definition & Modality \\
\hline \multicolumn{4}{|c|}{ Microstructure group } \\
\hline Ct.Po & $\%$ & pore volume fraction & $\mathrm{SR}-\mu \mathrm{CT}$ \\
\hline $\mathrm{PoS} / \mathrm{PoV}$ & $\mathrm{mm}^{-1}$ & pore surface to pore volume ratio & $\mathrm{SR}-\mu \mathrm{CT}$ \\
\hline $\mathrm{PoN}$ & $\mathrm{mm}^{-1}$ & pore number per millimeter & $\mathrm{SR}-\mu \mathrm{CT}$ \\
\hline PoDm & $\mu \mathrm{m}$ & average diameter of the pores & $\mathrm{SR}-\mu \mathrm{CT}$ \\
\hline PoSp & $\mu \mathrm{m}$ & average separation between pores & $\mathrm{SR}-\mu \mathrm{CT}$ \\
\hline PoPf & $\mathrm{mm}^{-1}$ & $\begin{array}{l}\text { pore pattern factor, lower } \mathrm{PoPf} \text { indicates } \\
\text { higher concavity, i.e., better-connected pore } \\
\text { network }\end{array}$ & $\mathrm{SR}-\mu \mathrm{CT}$ \\
\hline ConnD & $\mathrm{mm}^{-3}$ & $\begin{array}{l}\text { connectivity density, a measure of the degree } \\
\text { to which a pore is multiply connected }\end{array}$ & $\mathrm{SR}-\mu \mathrm{CT}$ \\
\hline SMI & a.u. & $\begin{array}{l}\text { structure model index, the relative prevalence } \\
\text { of rods and plates in a } 3 \mathrm{D} \text { pore network }\end{array}$ & $\mathrm{SR}-\mu \mathrm{CT}$ \\
\hline \multicolumn{4}{|c|}{ Mineral group } \\
\hline DMB & $\mathrm{g} / \mathrm{cm}^{3}$ & Degree of mineralization of bone & $\mathrm{SR}-\mu \mathrm{CT}$ \\
\hline MinOrga & no unit & $\begin{array}{l}\text { Mineral to organic ratio, the ratio of the } \\
\nu_{1} \nu_{3} \mathrm{PO}_{4} \text { area }\left(910-1184 \mathrm{~cm}^{-1}\right) \text { over the } \\
\text { Amide I area }\left(1592-1730 \mathrm{~cm}^{-1}\right)\end{array}$ & FTIRM \\
\hline MinMat & no unit & $\begin{array}{l}\text { Mineral maturity, the ratio of the ap- } \\
\text { atitic }\left(\sim 1030 \mathrm{~cm}^{-1} \text { peak }\right) \text { over non apatitic } \\
\left(\sim 1110 \mathrm{~cm}^{-1} \text { peak }\right)\end{array}$ & FTIRM \\
\hline Carbon & no unit & $\begin{array}{l}\text { Carbonation, the ratio of the } \nu_{2} \mathrm{CO}_{3} \text { area } \\
\left(862-894 \mathrm{~cm}^{-1}\right) \text { over the } \nu_{1} \nu_{3} \mathrm{PO}_{4} \text { area }\end{array}$ & FTIRM \\
\hline CryInd & $\mathrm{cm}$ & $\begin{array}{l}\text { Crystallinity index, the inverse of the full } \\
\text { width FTIRM at half maximum of the } \\
\sim 604 \mathrm{~cm}^{-1} \text { peak }\end{array}$ & FTIRM \\
\hline \multicolumn{4}{|c|}{ Collagen group } \\
\hline CollMat & no unit & $\begin{array}{l}\text { Collagen maturity, } \sim 1660 \mathrm{~cm}^{-1} \text { peak over } \\
\sim 1690 \mathrm{~cm}^{-1} \text { peak }\end{array}$ & FTIRM \\
\hline DHLNL & $\begin{array}{l}\mathrm{mmol} / \mathrm{mol} \text { colla- } \\
\text { gen }\end{array}$ & $\begin{array}{l}\text { Didhydroxylysinonorleucine, immature en- } \\
\text { zymatic cross-links }\end{array}$ & Biochemistry \\
\hline HLNL & $\begin{array}{l}\mathrm{mmol} / \mathrm{mol} \text { colla- } \\
\text { gen }\end{array}$ & $\begin{array}{l}\text { Hydroxylysinonorleucine, immature enzy- } \\
\text { matic cross-links }\end{array}$ & Biochemistry \\
\hline PYD & $\begin{array}{l}\mathrm{mmol} / \mathrm{mol} \text { colla- } \\
\text { gen }\end{array}$ & Pyridinoline, mature enzymatic cross-links & Biochemistry \\
\hline $\mathrm{DPD}$ & $\begin{array}{l}\text { mmol/mol colla- } \\
\text { gen }\end{array}$ & $\begin{array}{l}\text { Deoxypyridinoline, mature enzymatic } \\
\text { cross-links }\end{array}$ & Biochemistry \\
\hline PEN & $\begin{array}{l}\mathrm{mmol} / \mathrm{mol} \text { colla- } \\
\text { gen }\end{array}$ & Pentosidine, non-enzymatic cross-links & Biochemistry \\
\hline Coll & $\%$ & Collagen percentage by weight & Biochemistry \\
\hline
\end{tabular}

\section{Results}

\subsection{Descriptive statistics}

Microstructural, mineral and collagen variables and $Q_{44}^{-1}$ values have been obtained for 52 specimens. For each specimen measured by RUS, in average 5 values of $Q_{1}$ were successfully retrieved from the 6 available spectra. The average of these values, which was used for the analyses, ranged from 23.93 to 35.31 (30.07 \pm 2.02). The frequency $f_{1}$ of the first peak ranged from 115.4 to $160.3 \mathrm{kHz}(146.1 \pm 8.4 \mathrm{kHz})$. 
Table 2: Descriptive statistics (Mean $\pm \mathrm{SD}$ ) of $Q_{44}^{-1}$, microstructure and compositional variables. ${ }^{*}$ Variables in which significant difference were found between the data from lateral and medial specimens.

\begin{tabular}{|c|c|c|c|c|}
\hline \multicolumn{5}{|l|}{$\mathbf{Q}_{44}^{-1}$} \\
\hline \multicolumn{5}{|l|}{$0.0373 \pm 0.0031$} \\
\hline \multicolumn{5}{|c|}{ Microstructure variables } \\
\hline Ct.Po (\%) & $\mathrm{PoS} / \mathrm{PoV}\left(\mathrm{mm}^{-1}\right)$ & $\mathrm{PoN}\left(\mathrm{mm}^{-1}\right)$ & $\operatorname{PoDm}(\mu \mathrm{m})$ & $\operatorname{PoSp}(\mu \mathrm{m})$ \\
\hline $7.47 \pm 4.03$ & $60.53 \pm 18.29$ & $0.80 \pm 0.22$ & $89.39 \pm 31.98$ & $320.22 \pm 31.51$ \\
\hline \multirow[t]{2}{*}{$\operatorname{PoPf}\left(\mathrm{mm}^{-1}\right)$} & \multicolumn{2}{|c|}{ ConnD* $\left(\mathrm{mm}^{-3}\right)$} & \multicolumn{2}{|c|}{ SMI* $^{*}$ (a.u.) } \\
\hline & $\mathrm{L}$ & $\mathrm{M}$ & $\mathrm{L}$ & M \\
\hline $30.85 \pm 8.75$ & $10.32 \pm 4.99$ & $24.75 \pm 5.84$ & $3.23 \pm 0.25$ & $3.05 \pm 0.22$ \\
\hline \multicolumn{5}{|l|}{ Mineral variables } \\
\hline \multirow[t]{2}{*}{$\operatorname{DMB}\left(\mathrm{g} / \mathrm{cm}^{3}\right)$} & \multicolumn{2}{|c|}{ MinOrga* (n.u.) } & \multicolumn{2}{|c|}{ MinMat* (n.u.) } \\
\hline & $\mathrm{L}$ & $\mathrm{M}$ & $\mathrm{L}$ & $\mathrm{M}$ \\
\hline $1.02 \pm 0.02$ & $5.26 \pm 0.30$ & $5.55 \pm 0.26$ & $1.84 \pm 0.10$ & $1.72 \pm 0.07$ \\
\hline \multicolumn{2}{|c|}{ Carbon* (n.u.) } & \multicolumn{2}{|c|}{ CryInd* $^{*}(\mathrm{~cm})$} & \\
\hline $\mathrm{L}$ & $\mathrm{M}$ & $\mathrm{L}$ & $\mathrm{M}$ & \\
\hline $0.0071 \pm 0.0003$ & $0.0066 \pm 0.0002$ & $0.0384 \pm 0.0011$ & $0.0396 \pm 0.0006$ & \\
\hline \multicolumn{5}{|l|}{ Collagen variables } \\
\hline \multicolumn{2}{|c|}{$\begin{array}{c}\text { CollMat* } \\
\text { (n.u.) }\end{array}$} & $\begin{array}{l}\text { DHLNL } \\
\text { (mmol/mol } \\
\text { collagen) }\end{array}$ & $\begin{array}{l}\text { HLNL } \\
\text { (mmol/mol } \\
\text { collagen) }\end{array}$ & $\begin{array}{l}\text { PYD } \\
\text { (mmol/mol } \\
\text { collagen) }\end{array}$ \\
\hline $\mathrm{L}$ & M & & & \\
\hline $4.54 \pm 0.37$ & $4.33 \pm 0.29$ & $567.8 \pm 195.6$ & $260.4 \pm 73.4$ & $353.1 \pm 44.5$ \\
\hline $\begin{array}{l}\text { DPD } \\
(\mathrm{mmol} / \mathrm{mol} \\
\text { collagen })\end{array}$ & $\begin{array}{l}\mathrm{PEN} \\
(\mathrm{mmol} / \mathrm{mol} \\
\text { collagen })\end{array}$ & $\begin{array}{l}\text { Coll } \\
(\mathrm{mmol} / \mathrm{mol} \\
\text { collagen })\end{array}$ & & \\
\hline $103.1 \pm 18.3$ & $9.9 \pm 2.7$ & $13.5 \pm 0.7$ & & \\
\hline
\end{tabular}
in $[25]$ but is recalled here for the convenience of the reader.

$0.0373 \pm 0.0031$

Microstructure variables

Descriptive statistics of all variables are given in Table 2. Except for $Q_{44}^{-1}$, the data is the same as published

\subsection{Univariate correlation analysis}

Spearman rank correlation coefficients $(r)$ between $Q_{44}^{-1}$ and the other variables are summarized in Table 3. For the variables displaying a significant difference between the lateral and medial quadrants, i.e., ConnD, SMI, MinOrga, MinMat, Carbon, CryInd and CollMat, $r$ was calculated for lateral and medial group separately.

Among the microstructure variables, Ct.Po, PoN, and PoDm were positively correlated with $Q_{44}^{-1}, r$ was 0.68, 0.51 and 0.68 , respectively. Negative correlations were found between PoS/PoV, PoSp, PoPf and $Q_{44}^{-1}, r$ was $-0.68,-0.44$ and -0.61, respectively. Among the mineral variables, DMB was significantly correlated with $Q_{44}^{-1}(r=-0.68)$. Carbon from medial quadrant was significantly correlated with $Q_{44}^{-1}(r=0.40)$. Among the collagen variables, $Q_{44}^{-1}$, was weakly correlated with DHLNL $(r=0.32)$ and HLNL $(r=0.28)$.

The variables that are not significantly correlated with $Q_{44}^{-1}$, as shown in Table 3 , were not included in the subsequent regression analyses. 
Table 3: Spearman rank correlation coefficient $r$ between $Q_{44}^{-1}$ and microstructural properties. ${ }^{1} p<0.05,{ }^{2} p<0.01,{ }^{3} p<0.001$, n.s. not significant.

\begin{tabular}{|c|c|c|c|c|c|c|c|c|}
\hline \multicolumn{9}{|c|}{ Microstructure variables } \\
\hline & Ct.Po & PoS/PoV & PoN & PoDm & PoSp & PoPf & ConnD & SMI \\
\hline$r$ & $0.68^{3}$ & $-0.68^{3}$ & $0.51^{3}$ & $0.68^{3}$ & $-0.44^{2}$ & $-0.61^{3}$ & n.s. & n.s. \\
\hline \multicolumn{9}{|c|}{ Mineral variables } \\
\hline & \multirow[t]{2}{*}{ DMB } & \multirow[t]{2}{*}{ MinOrga } & \multirow[t]{2}{*}{ MinMat } & \multicolumn{2}{|c|}{ Carbon } & \multirow[t]{2}{*}{ CryInd } & & \\
\hline & & & & $\mathrm{L}$ & M & & & \\
\hline$r$ & $-0.68^{3}$ & n.s. & n.s. & n.s. & $0.40^{1}$ & n.s. & & \\
\hline \multicolumn{9}{|c|}{ Collagen variables } \\
\hline & CollMat & DHLNL & HLNL & PYD & $\mathrm{DPD}$ & PEN & Coll & \\
\hline$r$ & n.s. & $0.32^{1}$ & $0.28^{1}$ & n.s. & n.s. & n.s. & n.s. & \\
\hline
\end{tabular}

\subsection{Multivariate regression model}

In the multivariate regression models, $Q_{44}^{-1}$ is the dependent variable and the microstructure, mineral and collagen variables are the independent variables (Table 4). Overall, Ct.Po and DMB are the most significant factors contributing to the variability of $Q_{44}^{-1}$. As for the collagen variables, the only significant variable is DHLNL which only accounts for a minor part of the variability of $Q_{44}^{-1}\left(\mathrm{Adj}-r^{2}\right.$ is $5.7 \%$ ). In the multiple regression models using microstructure variables, Ct.Po explains most of the variations of $Q_{44}^{-1}\left(\mathrm{Adj}_{\mathrm{j}} r^{2}\right.$ is $53.2 \%$ ). Among the mineral variables, DMB is the most significant factor (Adj- $r^{2}$ is $43.2 \%$ ).

The most significant variable of each group, i.e. Ct.Po, DMB and DHLNL, was then retained to derive a multiple linear regression model. The result is a model with only two variables, Ct.Po and DMB, explaining $59.1 \%$ of the variability of $Q_{44}^{-1}$ (Table 4 ). The contribution of Ct.Po and DMB to $Q_{44}^{-1}$ are illustrated in Figure 6 .

Table 4: Multiple linear regression models of $Q_{44}^{-1}$. In the two-variable models, only Ct.Po and DMB are included. Note that the explanatory variables have been normalized . ${ }^{1} p<0.05,{ }^{3} p<0.0001$.

\begin{tabular}{|c|c|c|c|c|}
\hline Predicted variable & Explanatory variables & Linear model & $\begin{array}{r}\operatorname{Adj}-r^{2} \\
(\%)\end{array}$ & $R M S E$ \\
\hline$Q_{44}^{-1}$ & microstructure & $0.0396+0.0055 \times \overline{\mathrm{Ct} . \mathrm{Po}}$ & $53.2^{3}$ & 0.0021 \\
\hline$Q_{44}^{-1}$ & mineral & $0.0379-0.0051 \times \overline{\mathrm{DMB}}$ & $43.2^{3}$ & 0.0023 \\
\hline$Q_{44}^{-1}$ & collagen & $0.0377+0.0016 \times \overline{\mathrm{DHLNL}}$ & $5.7^{1}$ & 0.0030 \\
\hline$Q_{44}^{-1}$ & Ct.Po $+\mathrm{DMB}$ & $0.0392+0.0039 \times \overline{\mathrm{Ct} . \mathrm{Po}}-0.0025 \times \overline{\mathrm{DMB}}$ & $59.1^{3}$ & 0.0020 \\
\hline
\end{tabular}



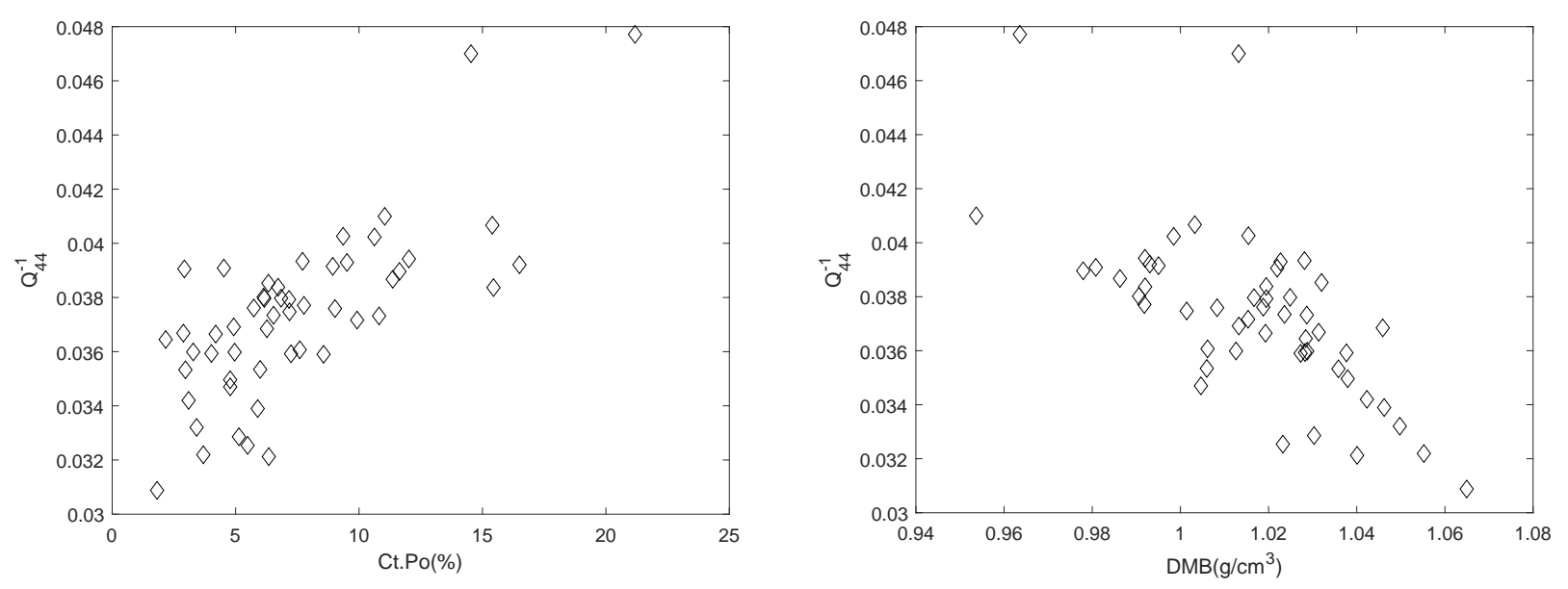

Figure 6: $Q_{44}^{-1}$ as a function of Ct.Po (left) and DMB (right)

\section{Discussion}

We measured with RUS the shear damping coefficient $Q_{44}^{-1}$, equivalent to a torsion loss tangent (usually denoted $\tan \delta$ ), in 52 specimens of human cortical bone from 26 elderly donors. We then investigated the relationships between $Q_{44}^{-1}$ and some compositional and microstructural characteristics measured with FTIRM, biochemical analysis, and SR- $\mu \mathrm{CT}$.

The shear damping values in the present study $(0.0371 \pm 0.0031)$ fall in the range of values usually reported [18]. The variations of $Q_{44}^{-1}$ were essentially determined by the variations of vascular porosity and mineral content: a multiple linear regression model with these two variables explained $59.1 \%$ of the variability of $Q_{44}^{-1}$. Damping increased with specimen's porosity and decreased with mineral content. Adding collagen variables did not improve this model. These relationships between damping, vascular porosity and mineral content have not been reported before as far as we know. These results are consistent with the finding previously reported [23] that $Q_{44}$ (also measured with RUS) is correlated to mass density $\left(r^{2}=0.72\right)$; indeed, density increases as porosity decreases and mineral content increases. Interestingly, this behavior for $Q_{44}^{-1}$ is similar to that observed for stiffness coefficients [25] which decrease with porosity and increase with mineral but are also weakly dependent on collagen variables.

Viscoelasticity in bone may arise from a variety of mechanisms, including fluid motion inside pores, thermoelastic coupling, motions at interfaces such as the cement line and between lamellae of mineralized collagen, and molecular deformation of collagen [3]. The relative importance of these mechanisms depend on the time scale of the experiment (or excitation frequency in a dynamic experiment). Garner et al. [18] have investigated the shear damping with an excitation frequency between $10^{-2}$ and $10^{5} \mathrm{~Hz}$, showing a minimum of damping around $10 \mathrm{~Hz}$ and a range of $\tan \delta$ of approximately 0.01-0.08. With RUS, the measurement frequency corresponds to the natural resonance of the specimen; in this study, this frequency varied in a narrow range centered at $146.1( \pm 8.4 \mathrm{kHz})$. Measurement of damping around $150 \mathrm{kHz}$ have seldom been reported as most of the viscoelastic data was obtained with dynamic mechanical analyzers (DMA) below $20 \mathrm{~Hz}$ or creep tests. Accordingly, our results can only be compared with that of others with caution. 
The relative importance of damping mechanisms also depends on the length scale of the measurement. Shepherd et al. [32] reported concurrent measurement of shear damping with a creep test on dogbone-shape specimens (several millimeters) and nanoindentation measurement of creep and found millimeter scale damping (relaxation time) about an order of magnitude larger than microscopic damping; furthermore the damping values at the two scales did not correlate. Accordingly, it is hypothesized that damping mechanisms, not captured by nanoindentation, dominate at the scale of a few millimeters: e.g., viscous damping related to fluid flow in pores, motion at mesoscale interfaces in the Haversian microstructure, or thermoelastic coupling at the mesoscale in the heterogeneous mineralized matrix. The correlation we found in the present study between porosity and damping is consistent with this hypothesis: with increased porosity the heterogeneity of the microstructure increases (leading to inhomogeneous thermoelastic damping) as well as the contact surface between fluid in pores and bone matrix (viscous damping due to fluid flow).

We built a multilinear model suggesting that when porosity is controlled, the variation of mineral content explains a small additional part of the variability of damping (Adj- $r^{2}$ of 59.1 vs. 53.2). Inter-specimen variation of mineral content may reflect different degree of homogeneity of the bone matrix (e.g., proportions of osteonal vs. interstitial tissue, age of osteons) which could affect damping through inhomogeneous thermoelastic effect and affect the viscous loss in nanoscale motion within the mineralized collagen fibrils [3]. At the scale of the mineralized collagen molecule, it was evidenced with molecular dynamics simulations that the mineral content contributes to the attenuation of stress waves [33]. The mineral characteristics other than DMB were weakly or non-significantly correlated to damping. Using nanoindentation and assessing mineral properties with FTIRM, Ojanen et al. [17] also found that mineral variables, except crystallinity, were not correlated to creep viscosity.

The role of matrix proteins in damping is well established [34, 35]. However, authors who have investigated the relationships between collagen and viscoelastic properties of native specimens (not chemically altered) reported weak or non significant correlations $[16,17]$. In line with these works, we found that collagen variables, including cross-links properties were weakly or non significantly correlated to $Q_{44}^{-1}$. One possible explanation is that the variations of collagen properties in the population of donors considered are too small and that at the macroscale, the variations of damping due to changes in microstructure are dominant.

Our data fills a gap of knowledge as we have measured cortical bone shear damping at frequencies around $150 \mathrm{kHz}$ which have seldom been considered [18]. This frequency regime is not accessible with most widespread measurement techniques: it is intermediate between the lower frequencies typically accessible with commercial DMA devices to measure the phase shift $\delta$ between a forced excitation and the oscillatory response, and higher frequencies ( $\mathrm{MHz}$ range) of conventional ultrasonic transducers to measure wave attenuation. The measurement frequency region considered is a priori not relevant for the study of the physiological behavior of bone as physiological loading hardly contains frequencies above $100 \mathrm{~Hz}$. However, some engineering applications could benefit from a better quantification of damping around $150 \mathrm{kHz}$. Low intensity pulsed ultrasound (LIPUS) devices used to stimulate bone healing work in a frequency range between $45 \mathrm{kHz}$ and $3 \mathrm{MHz}[36]$. Ultrasonic bone drilling in orthopedic surgery uses frequencies in the range $20-50 \mathrm{kHz}[37,38]$. Finally, some devices for the assessment of cortical bone properties for the monitoring of bone health with guided waves use frequencies around $100 \mathrm{kHz}[39,40]$. 
It has been suggested that viscoelasticity could serve as a biomarker of skeletal fragility and bone disease $[13,14,16]$. Most often, this idea is related to the assumption that interindividual variations of viscoelasticity reflect the variability in the quality of the bone matrix which should prevent crack propagation. Our results suggest that collagen variations only have a minor effect at the mesoscale (i.e., the scale of a few millimeters), if any, on damping variability. In contrast, we found that damping is correlated to porosity, which is a well documented risk factor for fragility fracture [41] and which is related to bone strength ex vivo [42]. This means that damping, as it reflects porosity, is related to bone fragility, supporting the consideration of viscoelasticity measurement as a potential biomarker of fragility.

This study has some limitations. The accuracy of shear damping measurement is limited by the fact that it was evaluated from the measurement of the quality factor of a resonance peak by using an approximate formulae (Eq. 5). We evaluated that only about $90 \%$ of the damping value was correctly captured. The resonance peak of the specimens ranged from 115.4 to $160.3 \mathrm{kHz}$ depending on the specimens dimensions, stiffness, and mass. We have disregarded a possible effect of frequency on shear damping independent of the effect of the microstructural and compositional variables. We believe this is reasonable as we did not find any correlation between the frequency of the peak and damping. Besides, bone specimens were collected at a single skeletal site (lateral and medial quadrants of the femoral diaphysis) of bones from elderly donors without documentation on the existence of bone pathologies. Therefore, the findings in this work are limited to the femoral mid-diaphysis of an aged group of donors. Further studies are warranted to investigate whether these conclusions can be extended to other skeletal sites of bone. Finally, studies with bone material representative of that of patients (e.g., osteopenic and osteoporotic patients) should be conducted in order to assess the extent to which viscoelasticity could reflect fragility for specific bone diseases.

\section{Declaration of interests}

The authors declare that they have no known competing financial interests or personal relationships that could have appeared to influence the work reported in this paper.

\section{Acknowlegement}

This work has received financial support from the Agence Nationale de la Recherche (France) under the project 418 ANR-13-BS09-0006 MULTIPS and China Scholarship Council under the file number 201806020131. Part of the work was performed within the framework of the LABEX PRIMES (ANR-11-LABX-0063) of the Université de Lyon, within the program "Investissements d'Avenir" (ANR-11-IDEX-0007). The authors thank the ESRF for allocated beam time within the experiments MD 927 on beam line ID 19 and MD 1056 on beam line ID 17. We also acknowledge the support of Lukas Helfen on beam line ID 19, as well as Herwig Requardt and Alberto Bravin on beam line ID 17. We also acknowledge Delphine Farlay and Evelyne Gineyts who performed FTIRM and biochemistry measurements, and Rémy Gauthier and David Mitton for help to prepare specimens. 


\section{Appendix A. Effect of the selected bandwidth for signal analysis on the peak frequency and quality factor.}

The quality factor of the first peak $Q_{1}$ in the RUS spectrum (Fig. 4) was determined by fitting the Lorentzian model, as explained in Methods, to a portion (bandwidth) of the spectrum containing one (Case 1) or two (Case 2) peaks. To test the effect of the choice of bandwidth we also fitted the spectrum with a bandwidth limited to the first peak for the 40 specimens initially in Case 2 (first two peaks relative close). Results are given in Table A.5. There was no significant difference for $Q_{1}$ and $f_{1}$ tested by Wilcoxon test. There was a slightly larger standard deviation of $Q_{1}$ calculated from the several repetitions of the measurements for each specimen due to the uptrend of the low frequency part of the second peak that was also taken into account. Overall, these results indicate that the bandwidth selection method has a negligible effect on the values of $f_{1}$ and $Q_{1}$ in this study.

Table A.5: Comparison of the results (mean $\pm \mathrm{SD}$ ) of $f_{1}$ and $Q_{1}$ obtained by fitting the first peak or alternatively the first two peaks. The last line summarizes the standard deviation (mean (SD)) of $Q_{1}$ calculated from the several repetitions of the measurements for each specimen.

\begin{tabular}{cc}
\hline $\mathrm{f}_{1}-$ Two peaks & $\mathrm{f}_{1}-$ One peak \\
\hline $145.9 \pm 6.5$ & $145.9 \pm 6.5$ \\
\hline $\mathrm{Q}_{1}-$ Two peaks & $\mathrm{Q}_{1}-$ One peak \\
\hline $29.98 \pm 1.81$ & $30.04 \pm 1.73$ \\
\hline SD of $\mathrm{Q}_{1}-$ Two peaks & $\mathrm{SD}$ of $\mathrm{Q}_{1}-$ One peak \\
\hline $1.04 \pm 0.70$ & $1.18 \pm 1.01$ \\
\hline
\end{tabular}

\section{Appendix B. Effect of defatting and X-ray radiation on viscoelastic damping}

In order to clarify whether $Q_{44}^{-1}$ would be affected by defatting and irradiation, RUS measurements were conducted three times on each specimen from a subset of 24 specimens: i) on the native specimen right after preparation, ii) after being defatted for $18 \mathrm{~h}$ in a chemical bath of diethyl ether and methanol (1:1), iii) after $\mathrm{SR}-\mu \mathrm{CT}$ imaging which delivers a moderate radiation dose of $2.5 \mathrm{kGy}$. More details concerning defatting and imaging protocols were given in [25]. Table B.6 summarizes the $Q_{44}^{-1}$ values determined from the three measurements.

Table B.6: A summary of the results $(\mathrm{Mean} \pm \mathrm{SD})$ of $\mathrm{Q}_{44}^{-1}$ in native, defatted and irradiated specimens.

\begin{tabular}{ccc}
\hline $\mathrm{Q}_{44}^{-1}-$ Native & $\mathrm{Q}_{44}^{-1}-$ Defatted & $\mathrm{Q}_{44}^{-1}$ - Irradiated \\
\hline $0.0375 \pm 0.0034$ & $0.0385 \pm 0.0031$ & $0.0378 \pm 0.0028$ \\
\hline
\end{tabular}

Multiple comparison of means did not show significant difference between the different states. There was no bias and the mean and standard deviation of differences appeared to be constant throughout the range of $Q_{44}^{-1}$ values. This analysis complements the analysis, conducted on the same specimens, of the effect of defatting and irradiation on elastic properties which was reported in [26]. 


\section{References}

[1] D. Huang, Y. Huang, Y. Xiao, X. Yang, H. Lin, G. Feng, X. Zhu, and X. Zhang, "Viscoelasticity in natural tissues and engineered scaffolds for tissue reconstruction," Acta Biomaterialia, vol. 97, pp. 74-92, 2019.

[2] R. Lakes, Viscoelastic materials. 2009.

[3] R. S. Lakes and J. L. Katz, "Viscoelastic properties of wet cortical bone - $\{\mathrm{II}\}$. Relaxation mechanisms," Journal of Biomechanics, vol. 12, no. 9, pp. 679-687, 1979.

[4] Q. Grimal, K. Raum, A. Gerisch, and P. Laugier, "A determination of the minimum sizes of representative volume elements for the prediction of cortical bone elastic properties," Biomechanics and Modeling in Mechanobiology, vol. 10, no. 6, pp. 925-937, 2011.

[5] D. B. Burr, "The contribution of the organic matrix to bone's material properties," Bone, vol. 31, no. 1, pp. 8-11, 2002.

[6] D. R. Carter and W. C. Hayes, "The compressive behavior of bone as a two-phase porous structure," Journal of Bone and Joint Surgery - Series A, vol. 59, no. 7, pp. 954-962, 1977.

[7] J. D. Currey, "Strain rate and mineral content in fracture models of bone," Journal of Orthopaedic Research, vol. 6, no. 1, pp. 32-38, 1988.

[8] R. Gauthier, H. Follet, M. Langer, S. Meille, J. Chevalier, F. Rongiéras, F. Peyrin, and D. Mitton, "Strain rate influence on human cortical bone toughness: A comparative study of four paired anatomical sites," Journal of the Mechanical Behavior of Biomedical Materials, vol. 71, pp. 223-230, 2017.

[9] Y. N. Yeni, R. R. Shaffer, K. C. Baker, X. N. Dong, M. J. Grimm, C. M. Les, and D. P. Fyhrie, "The effect of yield damage on the viscoelastic properties of cortical bone tissue as measured by dynamic mechanical analysis," Journal of Biomedical Materials Research. Part A, vol. 82, no. 3, pp. 530-537, 2007.

[10] R. A. Schapery, "A theory of crack initiation and growth in viscoelastic media $-\{$ I $\}$. Theoretical development," International Journal of Fracture, vol. 11, no. 1, pp. 141-159, 1975.

[11] R. O. Ritchie, M. J. Buehler, and P. Hansma, "Plasticity and toughness in bone," Physics Today, vol. 62, no. 6 , pp. $41-47,2009$.

[12] C. M. Les, J. L. Vance, G. T. Christopherson, A. S. Turner, G. W. Divine, and D. P. Fyhrie, "Longterm ovariectomy decreases ovine compact bone viscoelasticity," Journal of Orthopaedic Research, vol. 23, no. 4, pp. 869-876, 2005.

[13] X. Yang, S.-H. Teoh, S. DasDe, and T. Lee, "Administration of PTH and ibandronate increases ovariectomized rat compact bone viscoelasticity," Journal of the Mechanical Behavior of Biomedical Materialsr, vol. 22 , pp. 51-58, 2013. 
[14] X. Zhang, Z. Tang, Y. Zhou, X. Chen, X. Zhu, Y. Fan, Y. Zhang, X. Yang, C. qi Tu, and X. Zhang, “A multi-level comparative analysis of human femoral cortical bone quality in healthy cadavers and surgical safe margin of osteosarcoma patients," Journal of the Mechanical Behavior of Biomedical Materials, vol. 66, pp. 111-118, 2017.

[15] H. Isaksson, M. Malkiewicz, R. Nowak, H. J. Helminen, and J. S. Jurvelin, "Rabbit cortical bone tissue increases its elastic stiffness but becomes less viscoelastic with age," Bone, vol. 47, no. 6, pp. 1030-1038, 2010.

[16] Z. Wu, T. C. Ovaert, and G. L. Niebur, "Viscoelastic properties of human cortical bone tissue depend on gender and elastic modulus," Journal of Orthopaedic Research, vol. 30, no. 5, pp. 693-699, 2012.

[17] X. Ojanen, H. Isaksson, J. Töyräs, M. J. Turunen, M. K. Malo, A. Halvari, and J. S. Jurvelin, "Relationships between tissue composition and viscoelastic properties in human trabecular bone," Journal of Biomechanics, vol. 48, no. 2, pp. 269-275, 2015.

[18] E. Garner, R. Lakes, T. Lee, C. Swan, and R. Brand, "Viscoelastic dissipation in compact bone: implications for stress-induced fluid flow in bone," Journal of Biomechanical Engineering, vol. 122, no. 2, pp. $166-172,2000$.

[19] C. M. Les, C. A. Spence, J. L. Vance, G. T. Christopherson, B. Patel, A. S. Turner, G. W. Divine, and D. P. Fyhrie, "Determinants of ovine compact bone viscoelastic properties: effects of architecture, mineralization, and remodeling," Bone, vol. 35, pp. 729-738, 2004.

[20] A. Migliori and J. L. Sarrao, Resonant ultrasound spectroscopy. Wiley, New York, 1997.

[21] M. Radovic, M. W. Barsoum, A. Ganguly, T. Zhen, P. Finkel, S. R. Kalidindi, and E. Lara-Curzio, "On the elastic properties and mechanical damping of Ti3SiC2, Ti3GeC2, Ti3Si0.5Al0.5C2 and Ti2AlC in the 300-1573 K temperature range," Acta Materialia, vol. 54, no. 10, pp. 2757-2767, 2006.

[22] S. Bernard, Q. Grimal, and P. Laugier, "Resonant ultrasound spectroscopy for viscoelastic characterization of anisotropic attenuative solid materials," The Journal of the Acoustical Society of America, vol. 135, no. 5, pp. 2601-2613, 2014.

[23] S. Bernard, J. Schneider, P. Varga, P. Laugier, K. Raum, and Q. Grimal, "Elasticity-density and viscoelasticity-density relationships at the tibia mid-diaphysis assessed from resonant ultrasound spectroscopy measurements," Biomechanics and Modeling in Mechanobiology, vol. 15, no. 1, pp. 97-109, 2016.

[24] T. Lee, R. S. Lakes, and A. Lal, "Investigation of bovine bone by resonant ultrasound spectroscopy and transmission ultrasound," Biomechanics and Modeling in Mechanobiology, vol. 1, no. 2, pp. 165-175, 2002.

[25] X. Cai, H. Follet, L. Peralta, M. Gardegaront, D. Farlay, R. Gauthier, B. Yu, E. Gineyts, C. Olivier, M. Langer, A. Gourrier, D. Mitton, F. Peyrin, Q. Grimal, and P. Laugier, "Anisotropic elastic properties of human femoral cortical bone and relationships with composition and microstructure in elderly," Acta Biomaterialia, vol. 90, pp. 254-266, 2019. 
[26] X. Cai, L. Peralta, A. Giron, L. Helfen, C. Olivier, F. Peyrin, P. Laugier, and Q. Grimal, "Cortical bone elasticity measured by resonant ultrasound spectroscopy is not altered by defatting and synchrotron X-ray imaging," Journal of the Mechanical Behavior of Biomedical Materials, vol. 72, pp. 241-245, 2017.

[27] S. Bernard, G. Marrelec, P. Laugier, and Q. Grimal, "Bayesian normal modes identification and estimation of elastic coefficients in resonant ultrasound spectroscopy," Inverse problems, vol. 31, p. 65010, 2015.

[28] A. V. Lebedev, L. A. Ostrovskii, A. M. Sutin, I. A. Soustova, and P. A. Johnson, "Resonant acoustic Spectroscopy at low Q factors," Acoustical Physics, vol. 49, no. 1, pp. 81-87, 2003.

[29] Y. Sumino, I. Ohno, T. Goto, and M. Kumazawa, "Measurement of elastic constants and internal frictions on single-crystal MgO by rectangular parallelepiped resonance," Journal of Physics of the Earth, vol. 24, no. 3, pp. 263-273, 1976.

[30] H. Ogi, N. Nakamura, K. Sato, M. Hirao, and S. Uda, "Elastic, anelastic, and piezoelectric coefficients of langasite: resonance ultrasound spectroscopy with laser-Doppler interferometry," IEEE Transactions on Ultrasonics Ferroelectrics and Frequency Control, vol. 50, no. 5, pp. 553-560, 2003.

[31] R. G. Leisure, K. Foster, J. E. Hightower, and D. S. Agosta, "Internal friction studies by resonant ultrasound spectroscopy," Materials Science and Engineering: A, vol. 370, pp. 34-40, 2004.

[32] T. N. Shepherd, J. Zhang, T. C. Ovaert, R. K. Roeder, and G. L. Niebur, "Direct comparison of nanoindentation and macroscopic measurements of bone viscoelasticity," Journal of the Mechanical Behavior of Biomedical Materials, vol. 4, no. 8, pp. 2055-2062, 2011.

[33] M. Milazzo, G. S. Jung, S. Danti, and M. J. Buehler, "Mechanics of mineralized collagen fibrils upon transient loads," ACS nano, vol. 17, no. 7, pp. 8307-8316, 2020.

[34] N. Sasaki, Y. Nakayama, M. Yoshikawa, and A. Enyo, "Stress relaxation function of bone and bone collagen," Journal of Biomechanics, vol. 26, no. 12, pp. 1369-1376, 1993.

[35] M. Fois, A. Lamure, M. J. Fauran, and C. Lacabanne, "Study of human cortical bone and demineralized human cortical bone viscoelasticity," Journal of Applied Polymer Science, vol. 79, no. 14, pp. 2527-2533, 2001.

[36] F. Padilla, R. Puts, L. Vico, and K. Raum, "Stimulation of bone repair with ultrasound: A review of the possible mechanic effects," Ultrasonics, vol. 54, pp. 1125-1145, 2014.

[37] V. Gupta, P. M. Pandey, and V. V. Silberschmidt, "Rotary ultrasonic bone drilling: Improved pullout strength and reduced damage," Medical Engineering and Physics, vol. 41, pp. 1-8, 2017.

[38] A. Macbeath, Ultrasonic Bone Cutting. PhD thesis, University of Glasgow, 2006.

[39] P. Moilanen, M. Talmant, V. Bousson, P. H. F. Nicholson, S. Cheng, J. Timonen, and P. Laugier, "Ultrasonically determined thickness of long cortical bones: two-dimensional simulations of in vitro experiments," Journal of the Acoustical Society of America, vol. 122, no. 3, pp. 1818-1826, 2007. 
413

[40] A. Tatarinov, A. Sarvazyan, G. Beller, and D. Felsenberg, "Comparative Examination of Human Proximal Tibiae In Vitro by Ultrasonic Guided Waves and pQCT," Ultrasound in Medicine and Biology, vol. 37, no. 11, pp. 1791-1801, 2011.

[41] Y. Bala, R. Zebaze, and E. Seeman, "Role of cortical bone in bone fragility," Current Opinion in Rheumatology, vol. 27, no. 4, pp. 406-413, 2015.

[42] R. W. McCalden, J. A. McGeough, M. B. Barker, and C. M. Court-Brown, "Age-related changes in the tensile properties of cortical bone. The relative importance of changes in porosity, mineralization and microstructure," Journal of Bone and Joint Surgery - Series A, vol. 75, no. 8, pp. 1193-1205, 1993. 